\title{
Mutual Shaping in the Design of Socially Assistive Robots: A Case Study on Social Robots for Therapy
}

\author{
Katie Winkle ${ }^{1}$ (D) $\cdot$ Praminda Caleb-Solly $^{1} \cdot$ Ailie Turton $^{2} \cdot$ Paul Bremner $^{1}$
}

Accepted: 15 February 2019 / Published online: 7 March 2019

(c) The Author(s) 2019

\begin{abstract}
This paper offers a case study in undertaking a mutual shaping approach to the design of socially assistive robots. We consider the use of social robots in therapy, and we present our results regarding this application, but the approach is generalisable. Our methodology combines elements of user-centered and participatory design with a focus on mutual learning. We present it in full alongside a more general guide for application to other areas. This approach led to valuable results concerning mutual shaping effects and societal factors regarding the use of such robots early in the design process. We also measured a significant shift in participant robot acceptance pre-/post-study, demonstrating that our approach led to the two-way sharing and shaping of knowledge, ideas and acceptance.
\end{abstract}

Keywords Socially assistive robots · Robots in therapy $\cdot$ Robot acceptance $\cdot$ Design methods $\cdot$ Qualitative research methods

\section{Introduction}

Socially assistive robots (SARs) can be defined as those which provide assistance through social interaction alongside or instead of physical aid [9]. Researchers are increasingly exploring the use of such robots in the domains of healthcare and independent living (e.g. $[18,21,25,26])$. Research in these areas has shown that SARs can have an impact on enjoyment and engagement with health and wellbeing related exercises (e.g. $[13,16,32,35])$. We propose the use of a social robot to motivate and engage with rehabilitative therapy patients, and are working to identify the social cues and behaviours

Electronic supplementary material The online version of this article (https://doi.org/10.1007/s12369-019-00536-9) contains supplementary material, which is available to authorized users.

Katie Winkle

k.winkle@bristol.ac.uk

Praminda Caleb-Solly

Praminda.Caleb-solly@uwe.ac.uk

Ailie Turton

Ailie.Turton@uwe.ac.uk

Paul Bremner

paul.bremner@brl.ac.uk

1 Bristol Robotics Laboratory, Bristol, UK

2 Faculty of Health and Applied Sciences, University of the West of England, Bristol, UK such a robot should employ to enhance adherence to therapy. Low adherence to self-directed elements of therapeutic programmes is a known issue (e.g. [11,27,38]) and we believe that SARs could be useful in supporting these elements in order to improve it. Successful development and deployment of such robots requires, during the design stage, significant consideration of the social context into which they will be deployed. Robot use will influence, and be influenced, by this context. The general concept of technology and society influencing each other in parallel this way can be termed 'mutual shaping' [28]. We explored two forms of mutual shaping in a qualitative study with therapists as follows:

1. By working with the therapists to explore how deployment of SARs could change therapy delivery and influence/be influenced by the context of use.

2. By exploring mutual shaping during the study, considering how the study could influence the therapists' acceptance of SARs whilst generating useful information and perspectives through the process of mutual learning.

This paper presents our study methodology and our findings with regards to these objectives. We propose that focus groups following our methodology offer a tool for considering mutual shaping at three levels, as depicted in Fig. 1. In the first instance, taking part in the study influences participant acceptance and attitudes towards SARs. In addition, participants are able to identify social factors regarding the proposed 


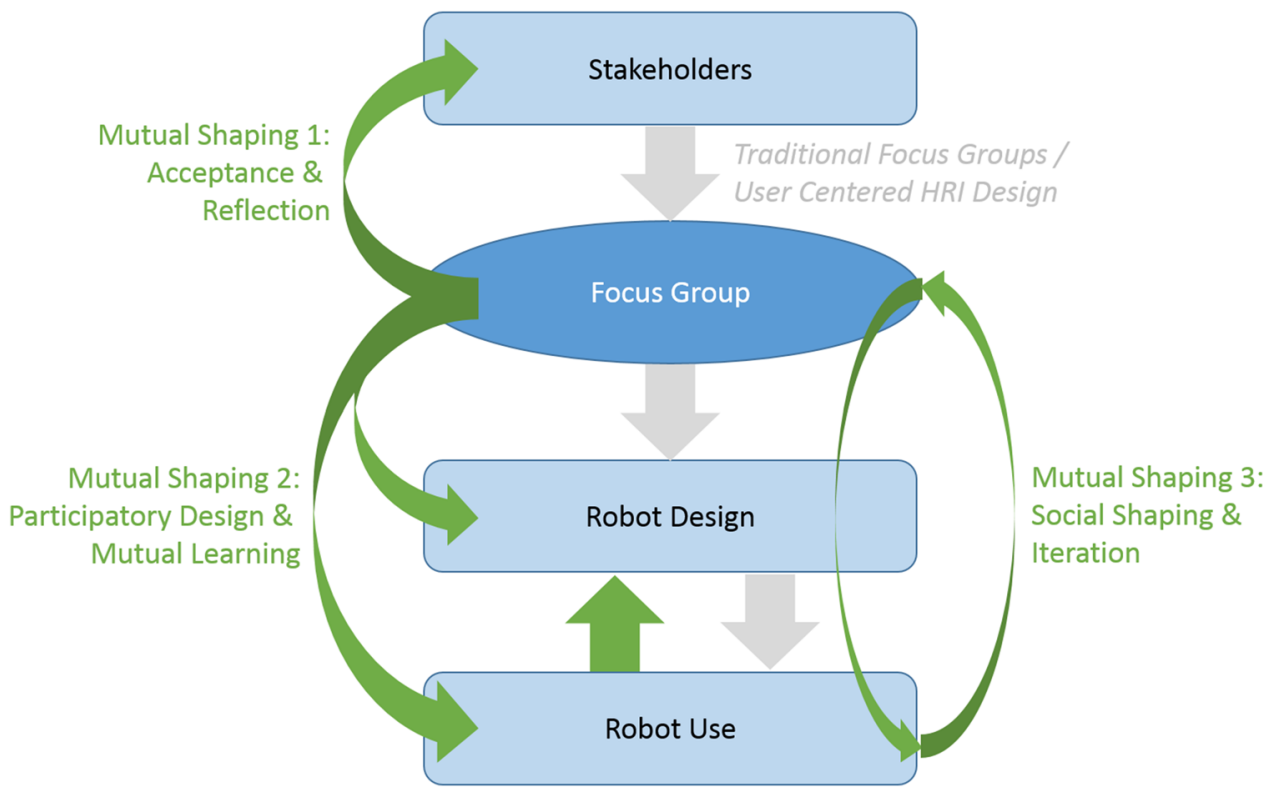

Fig. 1 Focus groups designed to include participatory design and mutual learning offer a mutual shaping tool for use as part of a mutual shaping approach to robot design. Alongside the traditional generation of user requirements and evaluation, they allow mutual shaping issues concerning real world deployment to be highlighted and discussed during the design stage (mutual shaping 2). In addition, they have

use case which are liable to influence effectiveness of the robot. This allows them to be considered during the design stage and hence reduces the risk of negative consequences on deployment. Both of these result from the process of mutual learning central to our design methodology. Finally, focus groups can be used throughout the design and deployment of SARs to allow for continued stakeholder input into design modifications that may result from real world or longer-term deployment. Detailed design guidelines for SARs in therapy resulting from this work are published elsewhere [43].

\subsection{Defining Mutual Shaping}

On investigating research practices in robotics, Sabanovic found that most roboticists take a technologically deterministic view of the interaction between robotics and society [28]. This view suggests that society should accept and adapt to robotic technologies, whose social impact is predominantly defined by their technological capabilities. However, studies in human-robot interaction have demonstrated that the impact of robots, when deployed in the real world, is influenced by a number of social and societal factors beyond this. For example, use and acceptance of a socially assistive robot, designed for health promotion to older adults, was found to be influenced by a diverse range of factors beyond just its practical functionality [8]. These included factors related to the context of use (e.g. social influence, privacy) and user characteristics (e.g. age, type of household). This example an impact on participant acceptance of SARs and engagement with the research (mutual shaping 1). Finally, we propose they can be used iteratively through the design and deployment of prototypes for investigating real-world and longer-term mutual shaping effects related to SAR use (mutual shaping 3)

demonstrates the impact of real world situational factors on use of the robot; however use of the robot can also influence and change such factors in return. For example, the PARO robot is primarily designed to reduce stress akin to animal therapy. ${ }^{1}$ Chang and Sabanovic undertook long term observations of PARO being used in a care home, using a social shaping framework to understand social factors that affected its use [6]. Similar to the previous study, they found that use of the robot was influenced by situational factors such as the users' gender, and that robot use was often prompted by another social actor (e.g. fellow resident, staff or visitor). In addition however, they also noted that use of the robot influenced staff approaches to care. For example a carer might move certain patients towards PARO when they would normally be guided elsewhere, or use PARO as a focal point during rehabilitation therapy sessions. This shaping of robot use by the social context and shaping of the use context by robot deployment can be described as 'mutual shaping'. In this article we primarily refer to mutual shaping issues around real world robot deployment such as these.

However, mutual shaping is not limited to use of a robot on deployment. Sabanovic proposed the framework of mutual shaping for social robot design, with a focus on the dynamic interaction between robotics and society at all stages of design, development and evaluation [28]. She proposed that one way to achieve this was to encourage user and stake-

\footnotetext{
${ }^{1}$ http://parorobots.com/.
} 
holder participation throughout the design and development process. In this article we also refer to the mutual shaping approach. Specifically we discuss how our methodology fits with this approach and why it is appropriate for considering, in advance of actual deployment, some of the mutual shaping issues around deployment as described above. Further, we also consider mutual shaping effects between researchers and participants resulting from taking such an approach, i.e. the impact that taking part in our study had on participants, as well as the information and perspective they were able to share with us.

\subsection{Mutual Shaping and Socially Assistive Robots}

SARs are typically designed to be used in socially complex applications with many stakeholders who may affect or be affected by their use. This could include teachers, parents, siblings, friends and classmates in the context of education, or carers, healthcare professionals, family and friends in the context of care; all representing stakeholders beyond the immediate user of the robot. Similarly the robot is likely to form part of a larger strategy or intervention, e.g. fitting in with a larger programme of study or forming one part of a care delivery plan. To be truly successful in the real world the robot needs to be accepted by all of these stakeholders. In our proposed use case, this means the robot must be accepted into both the patients' daily life and the therapists' approach to therapy delivery. Mutual shaping effects are inevitable in this scenario. Considered holistically they can represent an opportunity for making the best possible use of available technology. In contrast, failure to consider all stakeholders' views could lead to unpredicted negative consequences on real world deployment. As such, we suggest that a mutual shaping approach to the design of such robots is both appropriate and necessary for success. Further considering our use case specifically, the aim is that SARs should provide social support equivalent to that of a peer, therapist or family member, which has been shown to improve adherence to a therapy programme or exercise regime (e.g. $[4,27,41]$ ). To emulate such social support, the robot should be able to recognise and respond appropriately to the user's engagement, motivation, mood etc. just as human social support would. This requires an understanding of interaction context, cultural values and norms, the same factors relevant to the social shaping of technology [42]. As such, a mutual shaping approach which considers these factors is necessary for the design of effective and appropriate robot behaviours.

\subsection{Studying Conventional Therapy to Inform SAR Design}

An understanding of conventional therapy delivery is necessary if we are to pursue a mutual shaping design approach.
Therapists represent domain experts in our use case as well as stakeholders/end-users of our proposed system. Therefore, we propose to generate initial SAR functionalities and interaction behaviours based on their expert knowledge and working methods. We consider this part of a usercentered/participatory design approach as part of a mutual shaping framework for SAR development. This differs to other works on the development of other such SARs or chatbots which have typically utilised theoretical models from psychology (e.g. behaviour change theory [39]), ethnographic observations of human interactions [31] or machine learning (e.g. [5,23]).

\section{Related Work}

The framework of mutual shaping and co-production for social robot design was proposed by Sabanovic as an alternative to traditional approaches based on a technologically deterministic view of technology and society [28]. This approach proposes a bidirectional relationship between society and robotics, which must be considered during the robot design process if robots are to be successful outside of laboratory environments. Design recommendations for pursuing a mutual shaping approach include involving users in early stages of development, designing based on observation and empirical research (i.e. from the 'outside-in' [29]) and designing with users. Section 2.1 identifies key methodologies for mutual shaping, in order to properly situate our methodology with respect to e.g. user-centered and participatory design. Section 2.2 then reflects on the use of such methods in the design and development of SARs.

\subsection{Methods for Mutual Shaping}

There are a number of methodologies that might be employed in a mutual shaping approach to SAR design and research, and it is useful to define them in order to properly situate existing works. These include:

1. Ethnographic/'In the Wild' Studies typically focus on understanding situated use and/or emergent behaviour(s) on deployment of a robot into the real world. Concerning robot design, such studies are inherently limited to the testing of prototypes. However, they might be used to inform initial design requirements through observation of the current use case environment and user behaviour.

2. User-Centered Design aims to understand and incorporate user perspective and needs into robot design. Typically researchers set the research agenda based on prior assumptions regarding the context of use and proposed SAR application. 
3. Participatory Design encourages participants (users, stakeholders etc.) to actively join in decision making processes which shape robot design and/or the direction of research. This typically involves participants having equal authority as the researchers and designers, with both engaging in a two-way exchange of knowledge and ideas.

These terms define relatively high level methodologies or research philosophies. Specific data gathering methods which can be employed in pursuit of the above include focus groups and workshops (e.g. [19,22,24]), interviews [22], surveys [17] and observations (e.g. [6,12,30]). Lee et al. give a good overview of the above practices as currently employed in robot design, with a focus on user participation in the design process [22]. On the distinction between user-centered design and participatory design, they note that user-centered design typically tries to understand user needs for informing robot design. Participatory design instead attempts to empower participants such that they can actively collaborate in the design process. The authors also describe the concept of mutual learning, a key mechanism for achieving such collaboration. Via mutual learning, users learn about design and technology from the researchers as well as providing useful information and perspective to the researchers. This empowers the users to be able to really take part in the design process, giving them the knowledge required to conceptualise and hence critically evaluate the concepts proposed. Following these definitions, our work utilises elements of both usercentered and participatory design, with a focus on mutual learning.

\subsection{Mutual Shaping and Socially Assistive Robots}

Most studies concerning SARs in therapy have been concerned with feasibility and quantifiable impact (e.g. [16,33]) rather than exploring use cases, generating design recommendations or considering mutual shaping effects. Studies designed to measure user acceptance have also typically followed a technologically deterministic approach, with a complete system being presented for evaluation. For example, in the most closely related work considering SARs specifically for rehabilitation, Wilk and Johnson utilised a robot demonstration in investigating the potential for a combined telepresence/SAR system in facilitating and encouraging engagement with stroke therapy [40]. Residents and caregivers from a daycare centre were given a demonstration of the robot's capabilities. Then, they were asked to complete a survey measuring perception and acceptability of the robot system. The authors note that caregivers also discussed additional capabilities the robot could have, but no detail is given as to the format or formality of these dis- cussions. Further, there wasn't any consideration of, nor any opportunity to explore, mutual shaping effects that might arise through deployment of the system. Similarly, whilst caregivers identified potential robot applications, how the robot would be incorporated into overall care delivery was not discussed.

Considering SARs more generally, other research considering robots for the care of older adults has typically employed user-centered design to elicit user views or assess user needs for informing design requirements (e.g. [3,24, 44]). Whilst these works provide valuable user insight, they do not amount to pusuit of a mutual shaping approach because they fail either to i) account for the influence of social context on robot deployment and/ or ii) allow societal influence on robot design or research direction during the development stage. Other works however have specifically employed a mutual shaping approach, either through observing users and robots in real social environments via ethnographic studies. $[6,12,30])$ or through attempting to actively involve users in robot design [1,22] or the shaping of robotics research [19].

Specifically using participatory design, Azenkot et al. generated design specifications for a SAR that could guide blind people through a building [1]. The authors' study consisted of multiple sessions including interviews, a group workshop and individual user-robot sessions. Initial interviews were used to brief participants about the research and robot capabilities. The group session was used to develop a conceptual storyboard of robot use, identifying interactions between the robot guide and the user. Finally, participants were individually invited to work with a researcher and robot platform to prototype robot behaviour. The researchers also asked participants to instruct a naive human guide, asking probing questions around their preferences and instructions as a form of contextual inquiry attempting to elicit tacit knowledge.

Somewhere between user-centered and participatory design is Jenkins' and Draper's use of focus groups to explore views on care robots [19]. The authors used focus groups to collect views on ethical issues stemming from the real world deployment of care robots from older people and their carers. Participants were presented with pre-designed scenarios in order to prompt discussion, which may have limited the scope of discussions but ensured that the context of use was well established. This meant that the use of such robots was considered very holistically in terms of real world situations. In addition, participant responses were used to prompt discussion on how the SAR-integrated care approaches could be adjusted in order to make them more acceptable and reduce negative consequences on deployment. This work (and the larger research project from which it stems) fits somewhat with the mutual shaping approach in its attempt to consider SARs in care holistically and in allowing stakeholders to shape their work through revision of the pre-designed scenarios between iterations of the focus groups. Our work shares 
this aim of understanding the potential for mutual shaping on deployment early in the design process, in that we consider how therapy is currently delivered and how that would change due to use of SARs.

Particularly relevant to our work is Lee et al.'s use of participatory design methods in the development of SARs for older adults with depression [22]. The authors present a multi-stage participatory design process including interviews and workshops designed to facilitate co-design. They note that the use of multiple sessions allowed participants and researchers to 'familiarise themselves with each other's knowledge and build a relationship of trust'. Initial interviews were used to give researchers an understanding of the context of potential use (i.e. by visiting older adults in their home to see housing arrangements) and to encourage initial development of trust between the participants and the researchers. These were followed by a number of workshops with the older adults. The first workshop was used to introduce participants to examples of robot systems and to explore how they might be used. The second and third workshop focused on generating robot designs, after asking participants to reflect on some element of their life (challenges faced when lonely and use of space in the home respectively). The fourth workshop focused more on technical design with participants suggesting sensors that could be included in a robot and discussing resulting issues around data collection, sharing and security. Finally, a fifth workshop was used to share the robot designs generated by the older adults with therapists to get their perspective on such robots being used in the older adults homes. Our work shares Lee et al.'s focus on mutual learning and utilises the same aproach of introducing/explaining robot systems and getting participants to reflect generally on their current situation before encouraging participants to translate that into robot design requirements. However, our study aimed to achieve this in a single focus group session. Further, we also go beyond the generation of design input to examine the effects such an approach can have on participant acceptance of SARs.

The above literature demonstrates that whilst there is significant effort to include users in robot design, this is often achieved using user-centered design methods. Such methods typically focus on eliciting user perspectives in a one way exchange. Users provide information which designers and researchers then incorporate into robot design (e.g. $[3,24,44])$. Wu et al. note that their focus groups, designed to identify design preferences for robots for older adults, 'offered an opportunity for participants to...challenge some implicit preconceptions of the roboticists' [44]. One clear way we build on this is to additionally consider exactly the reverse; how roboticists can challenge participants preconceptions, attitudes towards and acceptance of SARs through a research study. None of the aforementioned studies do this, although Lee et al. do reflect on the potential participant ben- efits of participatory design (e.g. empowerment, increased social interaction) and how this should be considered in its use [22]. Similarly, even those studies specifically following a mutual shaping framework have typically been focused on the use of ethnographic studies to generate observation data and understand mutual shaping of robot use on deployment $[6,30]$ rather than during the design process, and hence given little opportunity for users or other stakeholders to voice their perspectives in SAR design. Works employing participatory design (e.g. [22] and [1]) are the exception, and demonstrate the worth of using such approaches.

\section{Methodology}

This study was undertaken as part of larger work to understand therapist-patient interaction and the potential for SAR use in therapy for informing design and development. The study design follows focus group methodology, as typically employed in user-centered design (e.g. [3,24,44]). Such focus group studies typically represent a one-way exchange with researchers simply looking to extract knowledge, information or ideas from the participants. Instead, we extend our use of focus groups toward more participatory design through use of mutual learning. We also investigate the impact of this approach on participant acceptance of SARs. Focus groups are an appropriate data collection method in the context of our research for two key reasons, as described in [2]. Firstly, they are good for accessing perspectives on novel topics. This is particularly relevant as the therapists we speak to are unlikely to have previously worked with or even considered the use of SARs in therapy. Secondly, they can facilitate the unpicking of thinking. This is important for understanding therapists' rationale e.g. in their approach to delivering therapy, their thoughts around how social robots could be helpful in therapy and their acceptance of SARs more generally.

We propose that our focus groups with therapists offer a valuable tool in pursuing a mutual shaping approach to SAR design and in the consideration of mutual shaping effects concerning real world robot use. Firstly, they allow the exploration of use case requirements and therapist best practice for achieving patient engagement. These can be used to inform the design of SARs to be used in this context (as per the traditional user-centered design). Secondly, the focus group can be used as an opportunity to educate therapists on state of the art technology and its intended uses, i.e. the research objectives of the project. In the first instance, this offers the chance to ease possible concerns regarding intended robot use and resulting impact (i.e. job losses, loss of human-human interaction etc.). Further however, it also allows for meaningful discussion on how social robots could aid in therapy delivery and how they might change current practice, based on participants having a better understanding of SAR capabilities. 
Finally, we predict that taking part in our focus group will have a positive influence on the therapists' attitude towards and acceptance of SARs in therapy. As such, we suggest our focus groups achieve mutual shaping by affording a two-way exchange of knowledge and ideas between participants and the researchers. Whilst we justify our approach with respect to our specific use case, it is easily transferable to other SAR applications and more general robotics research requiring input from stakeholders.

The aims of our study were as follows:

1. Inform social robot design and investigate robot acceptance (as per traditional user-centered design)

2. Follow a mutual shaping framework by considering:

(a) consequences and opportunities which may arise from real world robot deployment in this context

(b) how therapy programmes/delivery might change to make best use of SARs

(c) whether mutual learning employed in the study influences participant acceptance of and/or attitude towards SARs

The study consisted of 5 focus group sessions with therapists from a range of disciplines as shown in Table 1 (total pool $\mathrm{N}=19,2$ male and 17 female, average age 40). Therapists were recruited by email communications to local private practices, through advertising to university staff and through communications to contacts of the research team. Participants were randomly allocated to one of the sessions based on availability; group participant lists are given in Table 1 . All discussions were moderated (following guidelines from qualitative methods literature [2]) by the first author. The topic guide used by the moderator is presented in full in Online Resource 2. Sessions lasted between 60 and 100 minutes and were carried out at the Bristol Robotics Laboratory in the UK. Demographic information collected included time since qualified, time spent practising since qualified and typical service areas and users worked with. The study was approved by the ethics committee of the University of the West of England, Bristol.

Sessions consisted of discussions, a group activity, and a project talk with demonstrations using the robot Pepper ${ }^{2}$ given by the moderator. The ordering of these elements and key topics covered at each stage is shown in Table 2. Figure 2 shows the room layout employed for each session.

\subsection{Pre-focus Group Questionnaire}

We designed a set of questions to measure acceptance of social robots in therapy based on the Unified Theory

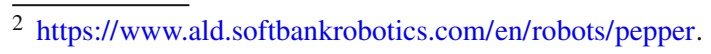

Table 1 Focus group participants in each group

\begin{tabular}{ll}
\hline Focus group & Participants \\
\hline 1 & OT1, OT2, OT3 \\
2 & SR1, P1, OT4, SL1 \\
3 & P2, P3, P4, P5 \\
4 & SL2, OT5, P6 \\
5 & SL3, P7, SR2, OT6, OT7 \\
\hline Participant coding: $O T$ occupational therapist, $P$ physiotherapist, $S L$ \\
speech and language therapist, $S R$ sports rehabilitation therapist
\end{tabular}

Table 2 Focus group schedule showing order of activities and related topics

\begin{tabular}{ll}
\hline Section & Key topics \\
\hline Before discussion & Acceptance questionnaire \\
Discussion part 1 & Use of social robots in therapy \\
& Conventional therapy delivery \\
& Group activity on factors affecting \\
& adherence \\
Study motivations \\
Project talk and demo & Supporting literature \\
& Project aims and objectives \\
& $2 \times$ Pepper assistance scenarios \\
& Revisit use of social robots in \\
Discussion part 2 & therapy \\
After discussion & Useful data collection \\
\end{tabular}

Note that the discussion is broken into two halves; pre and post the project talk and robot demonstrations

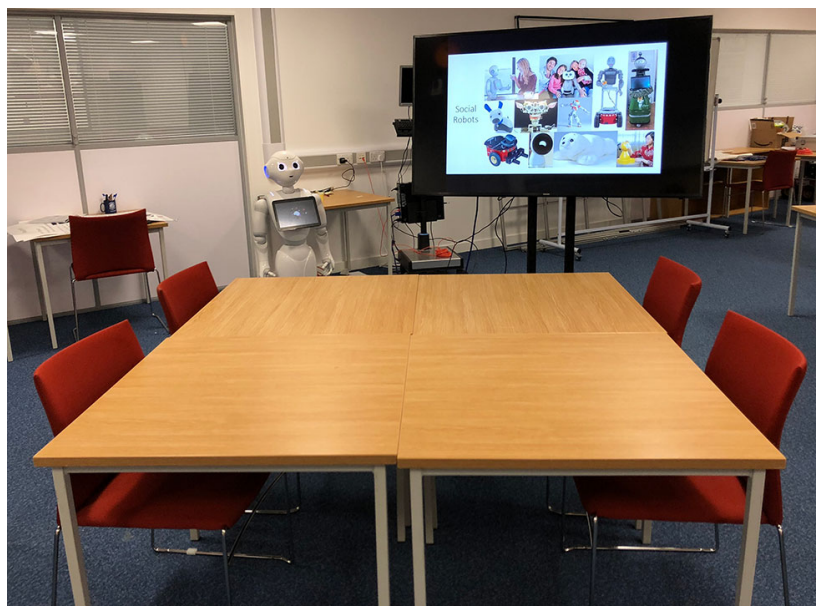

Fig. 2 Room layout during focus groups; note the presentation screen position on which a collage of different social robot images was displayed during discussions

of Acceptance and Use of Technology (UTAUT) [37]. A copy of the pre-focus group questionnaire is provided in Online Resource 1. The pre-focus group questionnaire was 
completed directly before the focus group began. The questionnaire asked participants to indicate their agreement with the following statements via a 5-point Likert scale (strongly disagree to strongly agree):

1. I feel apprehensive about the use of social robots in therapy

2. Social robots are somewhat intimidating to me

3. I think social robots might be somewhat intimidating to service users

4. I think using social robots in therapy is a good idea

5. I think using social robots would make therapy more engaging for the service user

6. A social robot would be useful in supporting a therapy programme

7. I think that use of a social robot could improve the positive outcomes/success of a therapy programme

Additionally a semantic difference question asked participants whether a social robot would be most useful when they were working with the patient or when the patient was working at home alone. We predicted that few participants would be familiar with the concept of social robots before participating in the focus group, potentially reducing the validity of questionnaire responses. To address this, we attached the pre-session questionnaire to the following definition of social robots (based on [10]): Social robots are those that can take part in social interactions with humans. They might exhibit human-social characteristics such as expressing and perceiving emotions, making conversation, establishing/maintaining social relationships, using natural cues (e.g. gaze, gesturing) and exhibiting a personality/character. Social robots might be humanoid/resemble some human characteristics but this is not always the case. A range of social robots are shown below to demonstrate this.

Alongside this description we added a collage of images containing 5 different social robots chosen from platforms known to the researcher (Pepper, Buddy, ${ }^{3}$ MiRo, ${ }^{4}$ Kismet $^{5}$ and Nao. ${ }^{6}$ )

\subsection{Pre-demonstration Discussion}

The pre-demonstration topic guide was designed to elicit participants' initial (relatively uninformed) ideas on the use of SARs in therapy. This covered ways in which SARs could be useful and more generally participants' feelings, attitudes

\footnotetext{
${ }^{3}$ http://www.bluefrogrobotics.com/en/home/.

4 http://consequentialrobotics.com/miro/.

${ }^{5}$ http://www.ai.mit.edu/projects/humanoid-robotics-group/kismet/ kismet.html.

${ }^{6}$ https://www.ald.softbankrobotics.com/en/robots/nao.
}

and/or concerns about their use, and was included to collect participants unbiased opinions. Contrasting this with postdemonstration discussion allowed for post-hoc investigation of mutual learning during the study. Specifically, participants were asked

\section{- What do you think about using robots to support a therapy program? \\ - How do you think that robots might be able to do that?}

See Online Resource 2 for full details of the topic guide.

During these discussions a collage of images of 10 different social robots (Pepper, Buddy, Kismet, Miro and Nao as previous, plus Bandit, ${ }^{7}$ Molly, ${ }^{8}$ Jibo, ${ }^{9}$ Paro, ${ }^{10}$ iCat $^{11}$ and Pioneer $^{12}$ (as used in Gockley and Mataric's related work as a 'social' robot [16]) was displayed on the presentation screen. This is shown in Fig. 2. Pepper was positioned alongside the moderator. Participants were able to ask the moderator questions concerning SARs, associated technologies or more about the project throughout the focus group. Pepper was positioned and set-up ahead of participants entering the focus group room; left visible and in its standard 'Autonomous Life' mode. This includes 'breathing' motion and the redirecting of gaze and body position based on visual human tracking. Audio input and output were disabled however; such that the robot was not speaking nor reacting to speech or sounds. The collage of social robot images was referred to by the moderator as appropriate during discussions, e.g. when faced with questions concerning physical design or to probe discussion points around capabilities and applications or participant reactions to Pepper.

This part of the discussion was also used to explore related conventional elements of therapy as they are done now; similar to the approach taken by Lee et al. [22]. This focused on the importance and implementation of self-practice exercises at home, e.g. therapist prescription, patient reporting and therapist monitoring of such exercises. Participants were also asked about their role in motivating or encouraging patients and how they might do that. Following this, participants were asked to complete a group activity ranking different factors which may affect patient exercise engagement. Participants were presented with a list of possible factors identified from the therapy literature, but also given blank cards to complete with any factors they additionally identified. An example result from this exercise is given in Fig. 3. As well as generat-

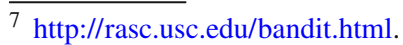

8 https://www.youtube.com/watch?v=nFZ9sUbbfe8.

9 https://www.jibo.com/.

${ }^{10} \mathrm{http} / / / \mathrm{www}$.parorobots.com/.

$11 \mathrm{https}: / / \mathrm{www}$.youtube.com/watch?v=7rCqclvf12Y.

12 http://www.mobilerobots.com/ResearchRobots/P3AT.aspx.
} 


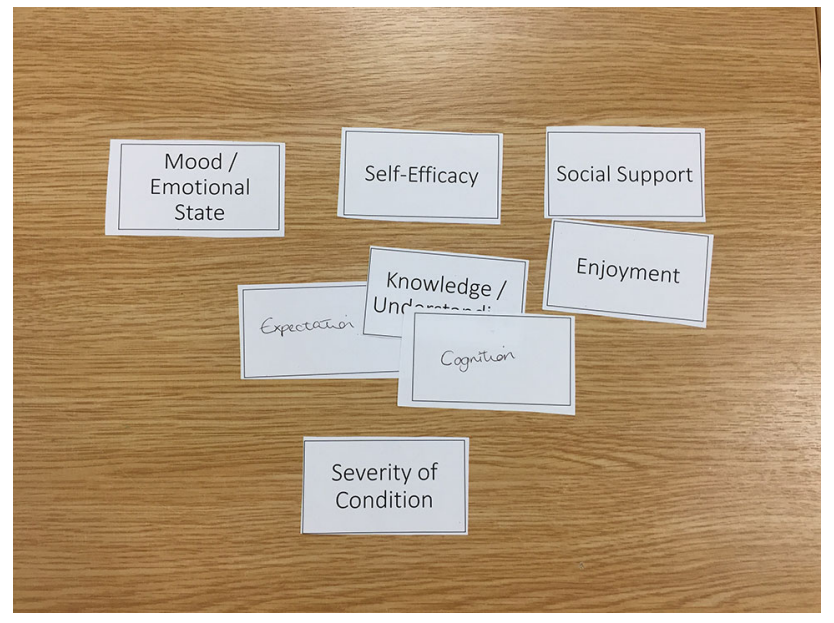

Fig. 3 Example output of the group ranking activity undertaken in the pre-demonstration discussion of focus groups. Participants were asked to order factors which might affect patient adherence to and engagement with exercises in terms of impact

ing data, this part of the discussion also established the focus group participants as 'experts'. Qualitative research guidelines [7] suggest that expert establishment in focus groups is key to encouraging participation. Participants then feel confident that they can offer useful, valid contributions and are therefore less hesitant to take part. All participants worked with self-practice of some form, and found 'common ground' on the need to motivate patients. This helped to set all participants equal and create rapport; again important for maximum participation.

\subsection{Project Presentation and Robot Demonstrations}

A key aim of the study was to achieve mutual learning between the researchers and the participants; as described by Lee et al. [22]. The project presentation and robot demonstrations represent a key mechanism for participant learning and the development of mutual trust (another key factor for participatory design). They were designed to (i) equip participants with a better understanding of socially assistive robotics and (ii) explaining the research aims and objectives, and the researchers' desire to work with therapists to design an appropriate and effective system. Following completion of the ranking exercise, participants were given a brief (approximately ten minute) presentation about the research project which covered:

1. Background robotics literature the moderator presented evidence suggesting that robots might positively influence engagement with therapeutic exercises (specifically highlighting [16] and [34]). Key points included i) embodied robots seems to have greater impact than a screens and ii) a robot which seems interested in what you're doing may encourage you to work for longer.

2. An overview of research aims, objectives and activities the moderator explained that this study was part of a larger research project exploring the use of SARs to encourage engagement in therapeutic exercises, lack of which is a known issue in rehabilitative therapies. It was explained that the ultimate aim of the project was to test a prototype with real patients, and that the aim of this study was to (i) generate some initial design guidelines and inspiration for that system based on the therapists' expert knowledge and (ii)further the the research team's understanding of conventional therapy delivery.

3. An introduction to the robot demonstrations it was made clear the the demonstrations were designed just to give an initial idea of what such a robot may 'look like' (including e.g. in terms of its behaviour and functionality). The moderator made a point of expressing her expertise as an engineer rather than a therapist in creating the demonstrations. However, it was noted that the demonstrations were designed in conjunction with an occupational therapy researcher (third author A Turton). The moderator also prompted participants to consider what they would have done as a demo themselves, what they would have liked to see and any 'can the robot...' questions they might have after watching the demo.

As discussed above, in the pre-demonstration discussions participants were initially asked very broadly how they thought SARs could be used in therapy. The presentation was then used as an opportunity to to share the researchers' proposed SAR application, i.e. for motivation and engagement in exercises in-between conventional therapy sessions. It was highlighted that such a system was not designed to replace the therapist. This is more in line with user-centered rather than participatory design, and is discussed further in the following subsection. However, the time taken to explain why and how the research team had identified that proposed application, i.e. the description of background literature described in the project presentation, was an attempt to foster mutual learning as per participatory design. Ahead of the demonstrations, participants were told that the demonstrations were very much a 'first draft' of possible robot behaviours designed only to illustrate the robot capabilities and give two examples of how such robots might be assistive in a therapeutic context. Afterward, participants were also reminded that the final aim of the research project was to produce more complex, personalised and responsive robot behaviours. As stated previously, up until this point Pepper operated in autonomous life mode but with audio input turned off. To initiate the demonstrations, audio input was re-enabled via a head touch, at which point the demonstrations were launched via voice command. After the demonstrations, Pepper was shut down manually 
using the chest button and displayed its standard shutdown behaviour animation.

The first demonstration showed Pepper facilitating a wrist exercise taken from a leaflet on Tennis Elbow produced by Arthritis Research UK. ${ }^{13}$ Pepper explained how to do the exercise with reference to images (taken from the Arthritis Research leaflet) displayed on its tablet. It then mimed checking the user's motion, counted repetitions for three sets of five exercises and gave encouragement. The second demonstration showed Pepper facilitating preparation of a microwave meal. Pepper gave step by step instructions and prompts, again with reference to images on the tablet. The demonstrations were live, with the moderator playing the role of a patient (undertaking the requested exercise, providing verbal responses of yes/no as appropriate etc.). Pepper was operating autonomously throughout the focus group, interacting with the moderator directly.

\subsection{Post-demonstration Discussion}

The presentation and demonstration made participants aware of our aim to use a SAR for motivation and engagement. The post-demonstration discussion was therefore more in line with user-centered design methods, with participants being invited to give feedback and perspectives on a predefined research agenda. This ensured that our focus groups still generated specific design requirements for our proposed application. Further, the data generated was improved by participants' increased understandings of SARs and their capabilities. Post-demonstration discussion centred on participants' reactions to the demonstrations and a revisit of the discussion concerning the use of social robots in therapy. Additionally, participants were asked whether there was any particular data about the patient that the robot could collect which would be useful for therapists to see. See Online Resource 2 for full details of the topic guide.

\subsection{Post-focus Group Questionnaire}

Directly after the focus group had been concluded and the audio recording equipment turned off, participants were asked to individually complete another questionnaire. This questionnaire contained a duplicate of the pre-session acceptance questionnaire (in order to investigate the impact of participation) plus some additional questions. These questions, designed to ensure maximum data capture and give a final opportunity for ideas and comments, were as follows:

1. In terms of achieving user behaviour change, what specific activities do you think a social robot could help with

\footnotetext{
$\overline{13}$ https://www.arthritisresearchuk.org/arthritis-information/ exercises-to-manage-pain/tennis-elbow-exercises.aspx.
}

and how? (e.g. activity prompts and feedback for exercises)

2. Which types of service user group(s) do you think could benefit from the use of a social robot?

3. Any other comments?

\subsection{Data Analysis}

Transcripts of the discussions were analysed using the Framework method, following published guidelines on the analysis of qualitative research data multi-disciplinary health research [14]. The data was first coded for key themes using a combined deductive and inductive approach to coding. Initial codes were generated based on the literature review and research questions. Two members of the research team then independently coded a transcript and generated any additional codes inductively as required. The researchers compared their individual codes (considering overlap, frequency of occurrence etc.) and a final coding scheme was generated for application to the transcripts. A second level of inductive coding was then applied to Applications of Social Robots in Therapy (before and after), Factors Influencing Engagement and Therapist Strategies for/Influence on Engagement in order to identify specific results. The resulting node diagram for Applications of Social Robots in Therapy is presented in Fig. 4 as an example. The full coding scheme (including all second and third level inductive codes) is given in Online Resource 3. Further according to the Framework method, themes were then identified across the data by reviewing the coded data excerpts and making connections within and between participants and coding nodes.

\section{Findings}

A key overall aim of this article is to demonstrate the value in taking a mutual shaping approach to the design of SARs, going beyond the general design requirements typically generated from qualitative design studies. Sections 4.1 and 4.2 therefore identify resulting insight concerning mutual shaping factors relevant to our proposed application; demonstrating that our approach resulted in the opportunity for these issues to be raised and discussed during the design process. These results should also be of interest for other researchers working on SARs for therapy. Sections 4.3 and 4.4 then demonstrate how our approach impacted participant SAR acceptance and engagement with the research.

\subsection{SARs in Therapy: Mutual Shaping Issues Identified for Consideration}

Across all focus groups participants identified a number of social and societal factors relevant to conventional therapy 
Fig. 4 Inductive coding nodes applied to data under

'Applications of Social Robots in Therapy'; identifying specific ways in which SARs might be useful in therapy and within that how they might target improved patient engagement

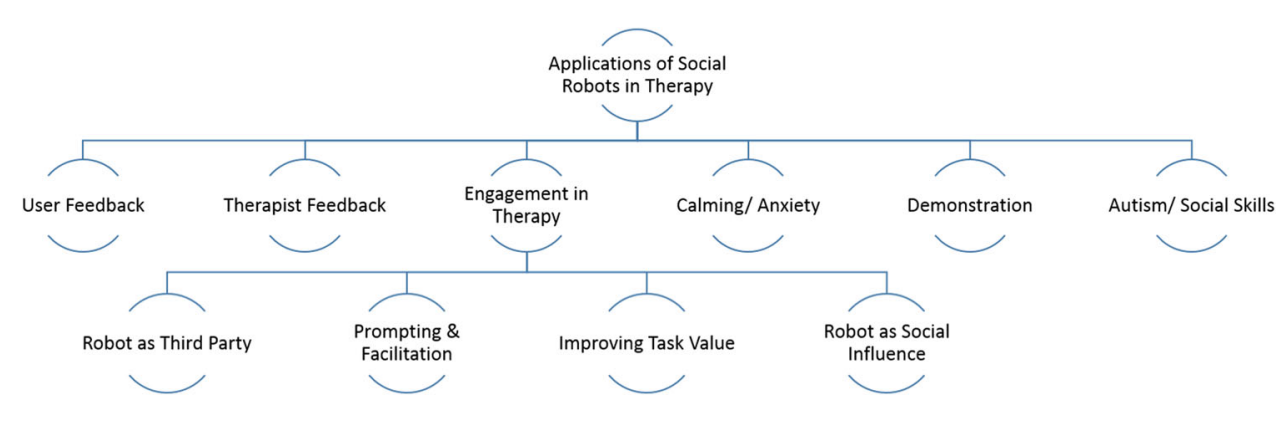

delivery. These particularly included factors which influence patient engagement and how the therapists worked to address that. Such factors are also likely to affect SARs deployed in therapy, and in some cases participants made this inference themselves. One key recurrent theme was the influence of (and potential to affect) the patient's immediate social circle, who are often a key support to both the patient and the therapist. A second key theme was the importance of the therapist-patient relationship and communication, both in terms of how this is crucial to conventional therapy delivery but also how SAR use might facilitate or be influenced by it. Another key theme was patient demographics and costing; although detailed financing was generally avoided by the moderator this raised some pertinent issues around patient socio-economic status with regard to therapy engagement. Finally, the potential for patients to become dependent on the robot was raised across 4 out of the 5 focus groups. These themes are discussed in more detail below.

\subsubsection{Issues Regarding the Patient's Social Life, Circle and Support}

The role of immediate family in encouraging the patient to engage was acknowledged multiple times:

His wife will always come back to me and say what he has and hasn't been able to do...she likes to make sure he's doing everything he's meant to do and sometimes he's sitting there and he's like 'well I tried to do it' (P2)

Some warned however that, particularly if the therapy was initially instigated or particularly encouraged by a particular family member, too much of this co-operation between the therapist and family could isolate the patient:

if the person around them is the one that's referred them and you're seen as that you're coercing, it's a bit of a conspiracy then isn't it and the person's going to feel a bit left out (SL1)

Some participants also noted the strain that therapy can put on personal relationships, and similarly whilst social support could be key in encouraging engagement, it could also have a negative impact as well: he's a good example of someone I've seen and given him exercises to do and challenges to do when I'm not there...he's got someone with him there who enables him to do it but he sometimes gets a little bit irritated because it's his wife (laughs)...And that's not uncommon either (P2)

sometimes the patient won't want to do the exercises with their for example husband because they know he's already doing all of the activities that they used to do and they don't want to be that additional burden 'they've already had a busy day I can't ask them to do my exercises with me so I just can't do my exercises (P5)

Participants were enthusiastic about the use of SARs to somehow address this, considering how SARs for therapy in the home could reduce carer load and relationship strain, but also raised potential concerns, e.g. the potential for guilt, that might be associated with that:

Parents, carers, they are absolutely knackered...we spend our lives telling them not to sit their children down in front of the telly to look after them so I think there might be, there's an issue of this around that as well like 'ooh I'm handing my child over to a machine to do what I should be doing'...I think it could help but there could be a guilt loop as well (SL3)

Such discussions generally focused on the SAR becoming a 'third-party'; i.e. something which could neutrally prompt the patient or alternatively be more convincing than a family member (especially in the case of young people):

Just thinking about I suppose the child-parent dynamic... actually the parent sometimes, you know, children say they're quite tired after school and parents like 'oh you don't need to do it tonight' or the opposite they try to get them to do it and they're like 'I'm not going to do that'. So actually with kids I think it could be a useful tool actually...a little bit more independent (OT4)

But then also a lot of people respond to a professional and not their family...So you could have the spouse sort of whistles to the robot 'Go on, give him a shout' (SL3) 


\subsubsection{How We Would, Could or Should Talk to Robots}

The concept of whether or not manners should be required or understood and responded to by a SAR was brought up both in terms of how the user would want to speak, but also about how that could affect or influence people around the robot:

I think bits of me like the human aspects but bits of me want it to almost to be a computer that talks (agreement from others) because I'm not sure... I would want to say please, and then I would hate myself for saying please to a machine (SL1)

I don't like speaking to Siri because it doesn't recognise when I say please, it then confuses it... when I'm talking in the car and my kids are hearing it I don't want them hearing it as if it's an instruction (OT7)

\subsubsection{Therapist-Patient Relationship and Communication}

All participants noted the important of the therapist-patient relationship, e.g. describing the importance of rapport and the influence that therapists can have on patients:

as therapists we've got the, well, sort of luxury of having time with people, so you do build up a relationship and mostly there's quite a lot of trust there and they put quite a lot of trust in what you say so you can be very influential with it (P2)

A suggested benefit of the system was addressing individual patient preferences with regard to monitoring and disclosure. For example, patients who didn't like reporting to the therapist could instead report to the robot, whereas those who seemed to benefit from therapist reinforcement could be reminded of that by the robot:

For some patients the idea that it's not a human that you're reporting to, and it would be faceless entity, could be a benefit to them, and them knowing that someone else is going to read it and observe it could be an issue for them... I would say it does make a difference because some of the technology that I use where it says about reporting the number of steps, they're waiting to be told off, even though you don't tell them off, or waiting to be praised. (P5)

One participant also noted that patients might be more willing to ask questions or ask for help via the robot based on previous experience with an email based system:

...what it also does is enable the patients to respond and write back in to their therapist with questions 'should I, shouldn't I, how do I do it' and in fact we have had a couple of patients who've been very engaged with it and even do so from their hospital bed and that was really interesting in terms of opening up and at what stage they share information and do those things and because there wasn't somebody there in front of them, which wasn't as off putting then actually you got very different information. (OT6)

\subsubsection{Robot Dependency}

Four participants independently referenced the risk of patients becoming dependent on the robot, having less autonomy or feeling less responsibility for managing their condition:

if she can go and get him a cup of tea then she would be loved. (OT3) That would be great (OT2) but at the same time your patients aren't getting mobile they're not getting up and aren't getting up and engaging. (OT3)

it's really important to keep the responsibility with the person themselves, which probably is going to be a factor with the robot as well you want to potentially be able to remove the robot from the situation at some stage (P6)

\subsubsection{Cost and Patient Demographics}

Given that cost is a typical barrier to adoption of new technologies, it was decided that issues relating to these aspects would not be discussed in depth. However, some participants highlighted more specific issues regarding cost. In the first instance, in the groups containing private practitioners, there were comments suggesting that patients who paid for their therapy directly were more likely to engage with their programme:

I find that people do the work more in private practice than they ever did in the NHS (SL3) But then, the fact that they are paying means that they have a vested interest (OT6)

In addition however, when considering factors which affect engagement (in which patient demographics was highlighted as a factor), some participants identified that those who might struggle with motivation most are least likely to be able to afford private therapy or related technologies:

I was at a sports medicine conference....and [Professor Greg Whyte] was talking about how actually interventions that we use to try and improve people's health through basically trying to improve their motivation to exercise...he's saying we're trying to solve sort of lower class problems with middle class, upper class solutions with things like apps and things you know...these are people who potentially don't have smart phones and yet 
all our efforts are being pointed at things like that technology which can't be afforded to these people (SR2)

\subsection{Willingness to Work With/Adapt to Using SARs}

During the pre-demonstration discussions, all participants indicated they would essentially be willing to try anything which might have a benefit for patients.

I think if you work with people, patients then anything that makes a positive difference preferably (laughs) is worth considering (OT6)

One participant offered an interesting reflection concerning what's best for therapists versus what's best for patients, and how those two things might unintentionally be misaligned, based on a previous experience working with technology in therapy:

I did a project quite some years ago about using video conferencing for face to face sessions and what was really interesting was that therapists were dead against it, including me, and felt that that would erode our one to one thing... and we really weren't very keen, people really weren't very keen, myself included. The patients loved it, so suddenly certainly for me I had to make a real leap into 'ok...I thought I was thinking of my patients' best interests but what I was actually thinking was about my satisfaction and the rewards for me' so I really had to change that and...so I'm probably coming at this much more positively than I perhaps would have done, having had that experience. (SL2)

One participant raised the issue that in the case of patients experiencing pain, the robot would have to tell them to stop, whereas a therapist would assess the situation and (if appropriate) reassure them that some pain is to be expected and suggest they should carry on. In response to this, another participant suggested this could be addressed by having the robot contact the therapist directly to facilitate this exchange as necessary:

you've also got to think about the questions that come along the route... the robot's just going to say 'stop you need to refer back to therapist', that sets up quite a sort of big message in the patients' brain of 'actually no I shouldn't be doing this because it hurts' and we might say 'oh its fine as long as its not making too much trouble for you, that's to be expected'... (P5)

But then you could have the robot doing that, so it could say 'ok sort of keep going, keep going for the moment, maybe not quite so strongly, let's message through to the therapist now'....and if the therapist gets a text that says this is what's going on and she can give you a call... it could be worked in (SL3)

Generally, participants who were less accepting or enthusiastic about the idea of using SARs were still able to identify ways in which they could be helpful; however these were more focused on fitting into current (conventional) therapy delivery. For example one participant expressed multiple doubts about robot technologies being suitable for occupational therapy (e.g. in their ability to 'read' the patient or be empathetic); however they were still able to identify how a robot might help to make better use of their own time with the patient:

I think you know the robot could help with something so if you say to the patient for next time prepare a list so that we can think about the groceries you need to do to be able to cook this meal or whatever it is I guess you know a robot or whatever could say have you prepared the list have you done this for tomorrow... so that sort of prompts that engagement into the task. (OT1)

In contrast, those that were more enthusiastic were able to come up with completely new applications and potential uses which the research team had not considered:

it's prompted me thinking of using some aspects of it for training other carers... a lot of the work I'm doing at the moment is teaching people in care homes working with people with dementia how to communicate with them... if you could show them how that works with a robot so 'this is what the robot is doing, isn't that more endearing when it does that, what would happen if you do that?' (SL1)

\subsection{Impact of Study Participation on Participants}

Our results suggest participant acceptance of SARs, and their potential for use in therapy, increased as a result of taking part in the focus group. Qualitatively this was evidenced by e.g. increasing positive comments and contribution to discussion. An example of this shift for one participant, who seemed to move from being very skeptical to more optimistic during the session, is given in Fig. 5. In-group discussion analysis identified that a concern or issue raised by one participant was sometimes responded to or addressed by another without moderator intervention. This was observed at least once in each focus group.

It doesn't go upstairs does it... so you'd have to have another one upstairs (OT2)

Potentially but that's something you can get over isn't it (OT3) 


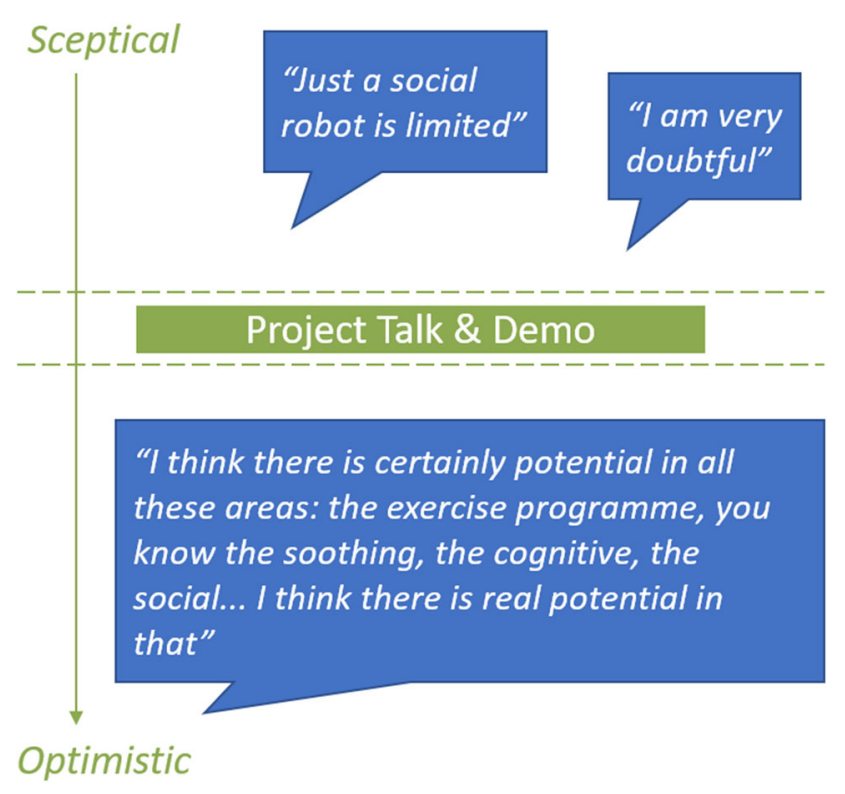

Fig. 5 Example shift in comments made by one participant in the pre and post demonstration discussions

But I mean it is a real issue then if someone's downstairs during the day... then they're upstairs for night time (OT2)

I' $m$ sure there is an adaptation you can stick on the bottom of the robot that she could get up the stairs (OT3)

The difference in participant acceptance before and after the study is also evidenced by the results of the pre/postsession questionnaire. The questionnaire results were quantified (using reverse coding where applicable) and a Wilcoxon Signed Rank Test was applied in order to measure the difference between the pre and post data sets for each acceptance statement. All statements except I think social robots might be somewhat intimidating to service users showed a significant $(p<0.05)$ increase in positive responses. The full results are listed in Table 3. The shift in acceptance is also shown visually for each statement in Fig. 6, which shows the spread of individual participant responses, and Fig. 7, which shows the shift in mean response across participants.

\subsection{Findings from the Process}

Following the impact on participants described previously, we also found that participants were generally very willing to be (and continue to be) part of the research. We believe this is due at least in part to the participatory nature of the focus groups, and the emphasis on allowing participants to shape the research. 17 of the 19 participants who took part in a focus group indicated they would be willing to take part in future studies, and to date we have been able to undertake 8
Table 3 Wilcoxon Signed Rank Test results comparing pre and postsession acceptance questionnaire; all except $*$ showing a significant increase in acceptance

\begin{tabular}{ll}
\hline Statement label & Result \\
\hline Apprehensive & $(\mathrm{Z}=-2.818, p=0.005)$ \\
Intimidating (me) & $(\mathrm{Z}=-2.309, p=0.021)$ \\
Intimidating (user) & $(\mathrm{Z}=1.811, p=0.70)^{*}$ \\
Good idea & $(\mathrm{Z}=-2.46, p=0.014)$ \\
More engaging & $(\mathrm{Z}=-3.116, p=0.002)$ \\
Useful & $(\mathrm{Z}=-3.0, p=0.003)$ \\
Improve success & $(\mathrm{Z}=-2.48, p=0.013)$ \\
\hline
\end{tabular}

interviews and 4 therapist-patient observations from this participant pool. Without further prompting, the first author has also since been sent relevant information (news stories, documentary recommendations etc.) on three different occasions by two different participants.

\section{Discussion}

\subsection{Mutual Shaping and Social Robots for Rehabilitative Therapies}

Our focus on mutual learning and move towards participatory design methods has helped to highlight a number of mutual shaping issues which should be considered in order to ensure successful deployment of SARs in therapy. These are summarised below. Note that this represents only an initial list which should be used to inform a next level of studies with other stakeholders (i.e. patients, carers etc.).

\subsubsection{Integrating SARs into Therapy Delivery}

Our results suggest that deploying a SAR for use in therapy requires adaptation of conventional therapy to be successful and have maximum benefit. This is true even for our application in which the robot would be used in-between standard sessions. Such adaptations could be universal (e.g. therapists being available for direct contact via the SAR in case of patient query) or individual to each therapist-patient pair. Participants were generally willing to consider adapting their working practices to accommodate SARs, and were able to offer practical solutions to issues arising from their deployment. This should be seen as great encouragement for researchers to undertake participatory design methods in the design and application of SARs. Participants also raised some potential long-term consequences arising from the deployment of SARs which need to be considered. These include the potential for reduced patient responsibility and autonomy, and improved communication via the robot as an intermedi- 
"I feel apprehensive about the use of robots in therapy"

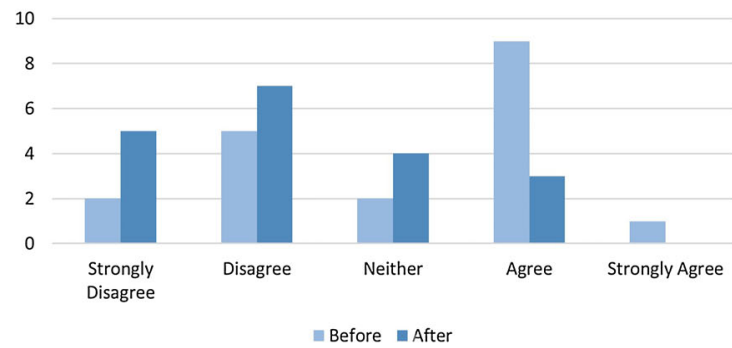

"I think social robots might be somewhat intimidating to service users"

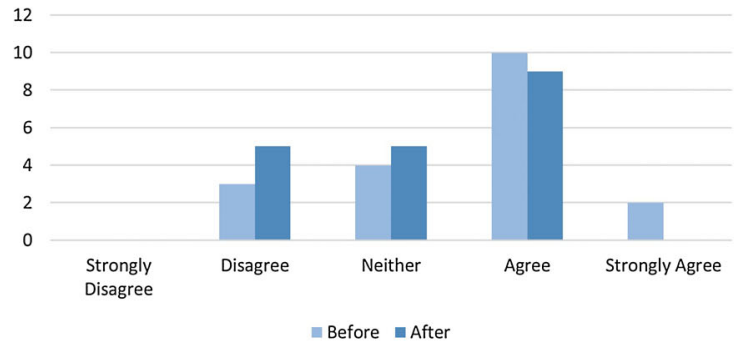

"I think using social robots would make therapy more engaging for the service user"

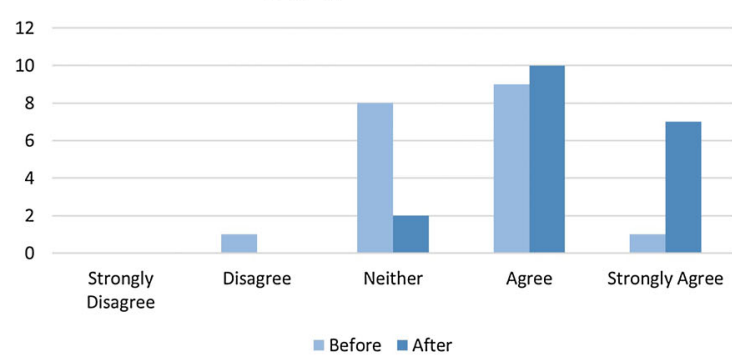

"I think that use of a social robot could improve the positive outcomes/success of a therapy programme"

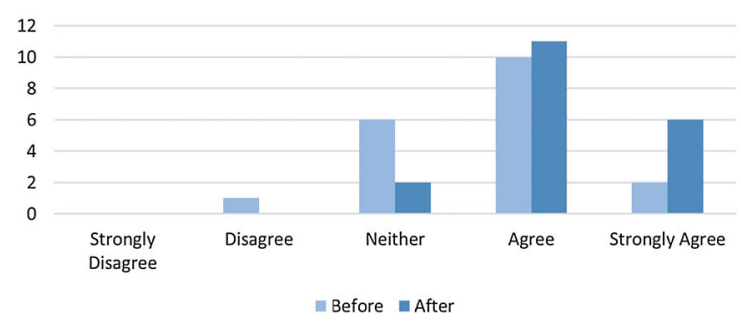

"Social robots are somewhat intimidating to me"

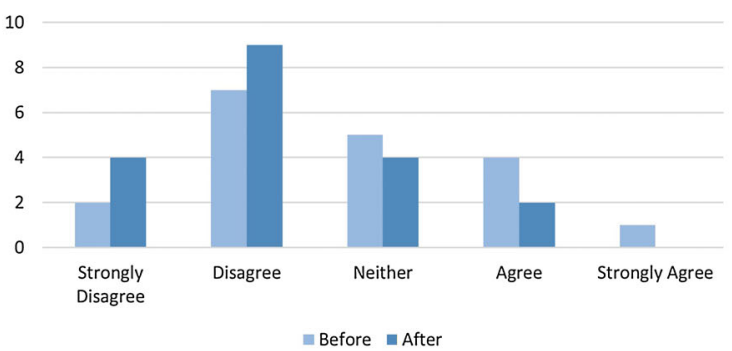

"I think using social robots in therapy is a good idea"

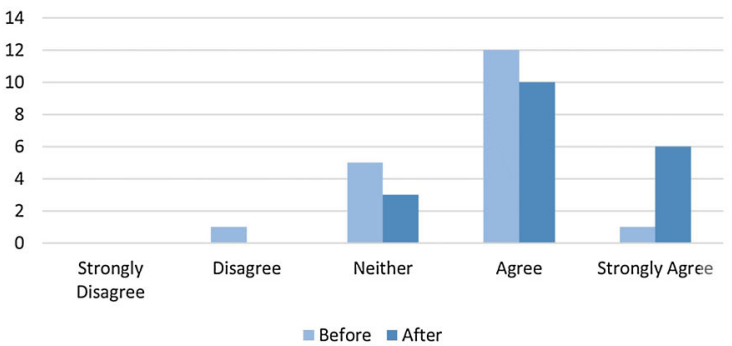

"A social robot would be useful in supporting a therapy programme"

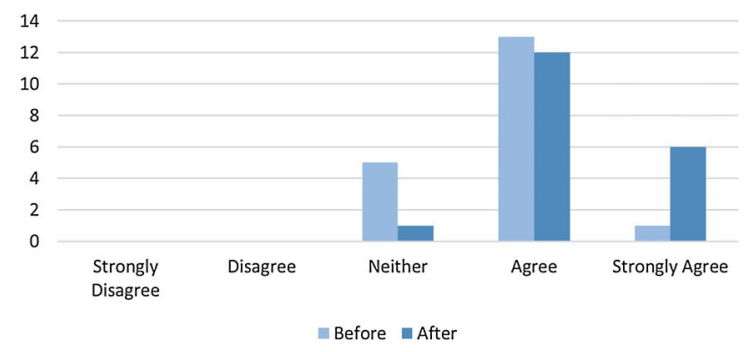

Fig. 6 Participant acceptance pre and post session questionnaire responses in full to show participant responses, their variation and the shift between questionnaires

ary. Such issues can only be investigated through realistic real world testing, i.e. tests which incorporate SAR use by the patient and associated adapted therapy delivery by the therapist.

\subsubsection{Impact of, and on, Patient Social Relationships}

Our results highlight the importance of considering people around the patient when deploying SARs. This must include recognising how such people, and their relationship with the patient, might affect robot use; but also how robot use can affect those people and impact on their relationships 
Fig. 7 Mean participant acceptance scores for the pre and post session questionnaires as another depiction of the shift between them

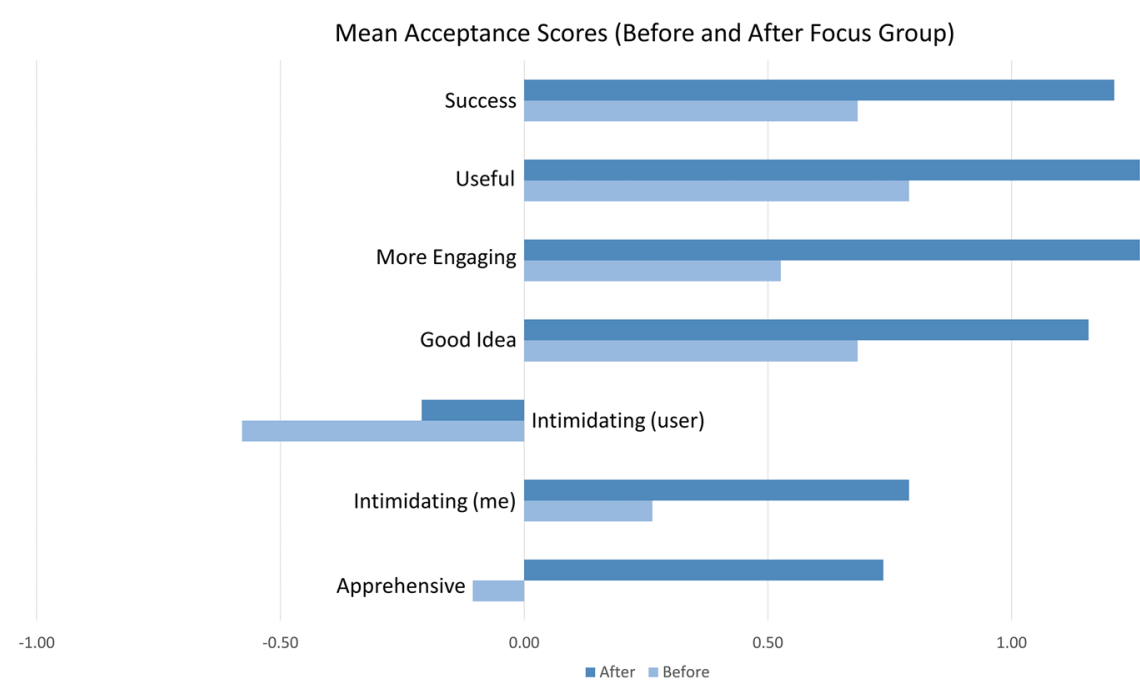

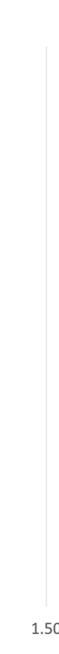

with each other. Whether those close to the patient (e.g. spouse, parents/children, friends) are accepting and encouraging about use of the SAR is very likely to have an impact on the patient's engagement with it. Conversely, the SAR should not be seen as a tool (brought in by the therapist and those people around the patient) responsible for enforcing compliance. In cases where the patient relies on care from immediate family and friends, SARs could represent a neutral third party which reduces tension around reminders and encouragement to exercises. However, this could also lead to feelings of carer guilt or replacement. These ideas and others around this topic require further investigation with patients and their families directly and require existential experience over a period of time.

\subsubsection{Demographic and Societal Factors}

Our results suggest that demographics and societal factors are inherently linked to patient engagement and therefore also important in considering the use of SARs in therapy. Participants highlighted that patients who pay for private therapy are somewhat motivated to engage based on that financial commitment. It could therefore be argued that any costs required to access a SAR system would be a barrier to those most in need of improved engagement. This further highlights the need to take a mutual shaping approach which considers new or revised therapy delivery methods that make use of SARs. It could be that such methods offer new ways of working with those patients who are currently either unable to access, or very difficult to engage in therapy.

\subsection{Participatory Focus Groups and Mutual Learning as a Mutual Shaping Tool}

Our results demonstrate that focus groups, as traditionally employed in user-centered design (e.g. $[3,24,44])$ can be extended to include an element of mutual learning and participatory design. We propose this fits with the mutual shaping approach to robot design proposed by Sabanovic [28]; and that our results demonstrate the worth of taking this approach. By combining user-centered and participatory design style discussions, we were able to generate specific design requirements for our application (presented in [43]) but also:

1. Identify a number of social and contextual issues likely to:

(a) affect real world use of a SAR on deployment

(b) be affected by such a deployment

2. Work with participants to identify how therapy delivery might be adapted to best utilise SARs, or at least in order to ensure successful real world use

3. Demonstrate that taking part in the study had a significant influence on participants' immediate acceptance of SARs in therapy

4. Establish a database of engaged therapists with an active interest in the research, i.e. willing to take part in future studies and share information with colleagues etc.

Based on the findings presented in Sect. 4 we hypothesise that participants felt empowered and authoritative after i) taking part in the participatory design element (pre-demonstration discussion) and ii) due to the project presentation which improved their understanding of SARs. The project presentation was also used to make it clear that participants' views were valued by the research team, likely increasing participant trust of the moderator. Participant empowerment was likely also achieved by having participants reflect on conventional therapy delivery, in which they are all inherently experts. This in turn allowed the postdemonstration discussions to be really grounded around the 
context of use, leading to the impressive generation of potential mutual shaping issues presented in Sect. 4.1.

We propose that our methodology could accommodate a mutual shaping approach to robot design at three levels, as depicted in Fig. 1 and summarised below. A guide to generalising our methodology is presented in Sect. 5.3.

\subsubsection{Stakeholder Acceptance and Reflection}

Mutual learning (i.e. which aims to also address participants' knowledge and understanding of the proposed robot and its purpose) has an immediate, positive impact on participant acceptance. This may also increase the chance of participants being willing to take part in future related research studies. Whilst individual focus groups target only small samples of the population, this methodology could be employed elsewhere, e.g. in user inductions on deployment. For example, if a number of therapists were about to be given a SAR to work with alongside their patients, something akin to our focus group with a focus on mutual learning might be delivered as a form of initial 'training'. Whilst this would not inform fundamental design of the SAR, it could inform its specific utilisation and modes of use by those specific therapists, hence improving acceptance and ensuring efficient utilisation in a way that works for that group.

The potential to improve acceptance and attitudes of therapists before they use the SAR could be hugely beneficial; especially given their position to influence patients (discussed in Sect. 4.1.3). Considering how mutual learning and study participation can influence participants goes beyond the typical one way flow of information from stakeholders to researchers normally seen in user-centered design. This is reflected in Fig. 1 with traditional focus groups taking information from stakeholders, but a mutual shaping approach having a return impact on those stakeholders such that the knowledge transfer is a two-way exchange.

Considering the impact on participant acceptance presented in Sect. 4.3, the biggest changes related to participants' apprehension about using SARs in therapy, whether SARs could make therapy more engaging for the patients and whether SARs could be useful in therapy. Whilst still significantly improved, the change in participant's finding SARs intimidating, agreeing the use of SARs in therapy was a good idea and that SARs could improve the success of therapy, was less. Figures 6 and 7 suggest that these statements were either already scored fairly positively in the pre-session questionnaire, or that participants' responses were more spread across the scale. Analysis of the focus group discussions suggests the reduction in apprehension could be linked to the robot demonstrations, which showcased how a SAR might actually look and behave. Participants seemed pleasantly surprised by Pepper's interaction abilities. Additionally it could be related to the project presentation which clarified the role of such robots and possibly reduced any concerns regarding job losses or replacement.

Concerning the increases on whether SARs could be useful and more engaging for patients, this is also likely to be due to participants' improved understanding of the proposed SAR usage and capabilities more generally. Most participants had never considered the use of social robots in therapy. Four of the physiotherapists mentioned manually assistive robotic rehabilitative devices ${ }^{14}$ they had seen or worked with and had initially been expecting to talk about. The only acceptance measure which showed no significant change was whether participants' felt that patients would find SARS intimidating. This is encouraging, as it suggests participants were clearly able to distinguish between their own feelings and their ideas of how patients might feel. In addition, it could suggest participants were reluctant to speak on behalf of patients. This correlates with comments during the focus group regarding patients all being individual, and suggestions that whilst some patients might love to work with a SAR, others might not want to at all.

\subsubsection{Participatory Design and Mutual Learning}

We propose that focus groups for informing robot design should be considered akin to participatory design sessions in which users can contribute to design ideas, rather than just e.g. evaluate pilot systems or rank specific design requirements. This is likely to improve both the eventual robot design and its success in the real world. Similarly, such focus groups should include an element of mutual learning in order to ground discussions and maximise the opportunity to identify mutual shaping effects concerning deployment. We moved towards participatory design by allowing participants to suggest uses of SARs before sharing our own research aims and proposed use. Then we used a project presentation to explain our research rationale and robot demonstrations to show our SAR capabilities and example uses as a form of mutual learning. Participatory design typically utilises multiple design sessions with participants (e.g. [1,22]) and whilst that is to be encouraged where possible and appropriate, our results suggest we were able to achieve similar benefits in a single session. By ensuring participants had a good understanding of robot capabilities and the context of use, we were able to generate detailed system requirements (presented in [43]) as well as the mutual shaping insights presented in this article.

\subsubsection{Social Shaping and Iteration}

We suggest that our focus group methodology is also appropriate for investigating mutual shaping effects arising from

\footnotetext{
${ }^{14}$ e.g. http://biorobotics.eng.uci.edu/armrehab.
} 
real-world and long-term deployment of SARs. Alongside ethnographic studies, focus groups should be employed iteratively during design and the deployment of prototypes. This would allow continued stakeholder input on design modifications and give insight to phenomena observed during observational studies. Focus groups could be run with naive or repeat participants. Working with repeat participants would mirror the multiple session studies employed in participatory design as discussed above. This would offer the to explore whether shifts in SAR acceptance resulting from the participation were long term. Further, whether that acceptance translates into real world usage behaviour. Such sessions would also benefit from established researcher-participant familiarity and trust. However, this same relationship and long-term engagement in the research could lead to increased Hawthorne effect [20] i.e. participants wishing to please the researcher and answer positively to suggest system success or improvement. Working with naive participants would avoid this, and could provide fresh ideas to the development process.

\subsection{General Methodology Guide}

We suggest our methodology is useful for SAR design across any application, as well as more general robotics research where user, stakeholder or non-expert perspective is valued. As a general guide, the key elements required to replicate our focus group approach are as follows:

1. Establish participants as experts and engage in broad discussion without presenting a defined research agenda (as per participatory design methods)

2. Get participants to reflect on the context of use as it is now; i.e. before introduction of a robot, in order to ground discussions

3. take time (in the middle of the session to allow for item 1) to then explain the research agenda (and motive) in more detail, as well as to share relevant technical expertise to improve participant understanding of the proposed technology/application. A demonstration would be useful here.

4. Revisit key topics of discussion after the above in order to get informed (and/or altered) opinions as well as any new ideas (as per user-centered design methods)

\subsection{Novelty Effect}

As most focus group participants had very little previous experience with robots, the well known 'novelty effect' phenomena (e.g. [15]) might have influenced their responses to the demonstrations given. We attempted to minimise this by explaining the basic concept of social robots alongside the initial acceptance questionnaire as discussed in Sect. 3.1. Further, it seems unlikely that novelty effect alone is responsible for the significant change in acceptance demonstrated; particularly given that it varied across participants. Participant comments link the change more with the project presentation, giving reasons such as understanding the research aims. However, this does give further reason for iteratively repeating such studies throughout the design process as discussed previously. Acceptance could then be periodically re-considered as participants become more familiar with, and start to use, the system in increasingly realistic settings. This could be done in part by re-administering a UTAUT [37] based questionnaire such as ours or similar (e.g the technology acceptance model [36]) which might be more appropriate for longer term testing. However, ethnographic observations and objective measures of robot utilisation should also be employed at this stage.

\section{Conclusion}

Socially assistive robots (SARs) are increasingly employed in socially complex domains such as healthcare and independent living. Whilst much work is being done to consider user acceptance of proposed SAR technologies, very few published research studies invite users to get truly involved in the design process. In addition, there is a trend for studies with users to be seen as one way exchanges with researchers learning and gaining insight from the users, with no consideration of the reverse. This paper seeks to demonstrate that focus groups, typically employed in user-centered design, can be extended to include elements of participatory design and mutual learning to address this. We propose such focus groups represent a tool for taking a mutual shaping approach to SAR design, and can be used to:

1. Positively influence participant acceptance of SARs by improving their understanding around capability and intended use

2. Provide guidance on how the proposed SAR will fit into existing assistance mechanisms and how these might be adapted to make best use of the SAR, thus maximising benefit and reducing the potential for negative consequences on deployment

3. Help surface additional mutual shaping effects (e.g. social and societal factors) linked to real world deployment and use of the intended SAR system

We have presented our method for structuring focus groups to combine elements of user-centered and participatory design and a focus on mutual learning to support a mutual shaping approach as intrinsic to their process. The 
rich set of findings elicited from our focus groups are evidence of the value of this approach. Our study consisted of 5 focus groups with a total of 19 participants, and was designed following guidelines for qualitative methods literature. Focus groups included a short presentation about the research project and two demonstrations of the Pepper robot. Participant acceptance was measured quantitatively before and after participation through a questionnaire as well as qualitatively based on analysis of the discussion and how it progressed during the focus groups, particularly comparing that before and after the presentation and robot demonstrations. The key outcomes of our study were as follows:

1. A number of ideas on how rehabilitative therapy delivery could or should be adapted to make best use of SARs (see [43] for detailed resultant design guidelines)

2. Identification of a number of social and contextual factors likely to affect/be affected by use of a SAR in the proposed application

3. Demonstrably improved participant acceptance of SARs and their use in therapy

4. High and continuing participant engagement with, and interest in, our overall research project

We conclude that focus groups can be a useful tool for more than just informing SAR design and eliciting expert knowledge. We find that they can also be used as a mutual shaping tool to influence key stakeholders and meaningfully explore mutual shaping effects through a two-way sharing and shaping of knowledge, ideas and acceptance. Our results should be of particular interest to those working on SARs for therapy, but our methodology can be applied to design and development of any robot intended for us in complex social scenarios.

\subsection{Limitations}

This paper presents initial findings from a first stage study with therapists. The work presented forms part of a larger, mixed methods research project on the use of SARs in therapy. We recognise that we have not yet significantly addressed mutual shaping effects around how SARs in therapy might impact the lifestyle of users. We hope to do this in the future through longitudinal, real-world human robot interaction experiments with our proposed SAR system. Further, as discussed in Sect. 5.2.3, we recognise that mutual shaping is a continuing and concurrent process and hence requires consideration and investigation even after initial SAR design and implementation. Continuing such work and iteratively updating SAR technology in response to the results is likely to be vital in ensuring such systems have maximum positive impact and real-world usability long term.
This work was limited to a study with therapists. It is intended that the results should inform preliminary interaction strategy design for first stage testing and meaningful co-design sessions with real end-users (patients and their therapists). As such, this work does not represent a longterm consideration of mutual shaping effects surrounding the use of SARs in therapy. Additionally, we identify the need to carry out ethnographic observation studies of therapistpatient interactions in order to assess the more real-time and tacit elements of interaction. This is also necessary to further achieve the outside-in design approach discussed in Sect. 1.3. The results presented here are also limited by participants' exposure to and understanding of SAR technologies and their exposure to a single platform. The impact of the 'novelty effect' is discussed in detail in Sect. 5.4. In summary, this work presents compelling evidence for taking a mutual shaping approach to SAR design, and offers a structured approach to conducting focus groups as part of a mutual shaping approach to design.

\section{Compliance with Ethical Standards}

Conflict of interest The authors declare that they have no conflict of interest.

Funding This study was funded by the EPSRC via the Centre for Doctoral Training in Future Autonomous and Robotic Systems (FARSCOPE) at the Bristol Robotics Laboratory, University of the West of England and University of Bristol (Grant Number EP/L015293/1).

Open Access This article is distributed under the terms of the Creative Commons Attribution 4.0 International License (http://creativecomm ons.org/licenses/by/4.0/), which permits unrestricted use, distribution, and reproduction in any medium, provided you give appropriate credit to the original author(s) and the source, provide a link to the Creative Commons license, and indicate if changes were made.

\section{References}

1. Azenkot S, Feng C, Cakmak M (2016) Enabling building service robots to guide blind people a participatory design approach. In: 2016 11th ACM/IEEE international conference on humanrobot interaction (HRI), pp 3-10. https://doi.org/10.1109/HRI. 2016.7451727

2. Barbour R (2008) Doing Focus Groups. SAGE (2008). GoogleBooks-ID: TzZTCEAK6N4C

3. Beer JM, Smarr CA, Chen TL, Prakash A, Mitzner TL, Kemp CC, Rogers WA (2012) The domesticated robot: design guidelines for assisting older adults to age in place. In: Proceedings of the seventh annual ACM/IEEE international conference on humanrobot interaction, HRI '12, pp 335-342. ACM, New York, NY, USA. https://doi.org/10.1145/2157689.2157806

4. Carron AV, Hausenblas HA, Mack D (1996) Social influence and exercise: a meta-analysis. J Sport Exercise Psychol 18(1):1-16. https://doi.org/10.1123/jsep.18.1.1 
5. Chan J, Nejat G (2012) Social intelligence for a robot engaging people in cognitive training activities. Int J Adv Robot Syst 9(4):113. https://doi.org/10.5772/51171

6. Chang WL, Šabanović S (2015) Interaction expands function: social shaping of the therapeutic robot PARO in a nursing home. In: Proceedings of the tenth annual ACM/IEEE international conference on human-robot interaction, HRI '15, pp 343-350. ACM, New York, NY, USA. https://doi.org/10.1145/2696454.2696472

7. Curry L (2015) Fundamentals of qualitative research methods. https://www.youtube.com/watch?v=wbdN_sLW188\& list=PLqHnHG5X2PXCsCMyN3_EzugAF7GKN2poQ. Accessed 1 Feb 2017

8. de Graaf MMA, Allouch SB, Klamer T (2015) Sharing a life with Harvey: exploring the acceptance of and relationship-building with a social robot. Comput Hum Behav 43:1-14. https://doi.org/10. 1016/j.chb.2014.10.030

9. Feil-Seifer D, Matarić MJ (2005) Defining socially assistive robotics. In: 9th International conference on rehabilitation robotics, 2005. ICORR 2005, pp 465-468. IEEE

10. Fong T, Nourbakhsh I, Dautenhahn K (2003) A survey of socially interactive robots. Robot Auton Syst 42(3-4):143-166. https://doi. org/10.1016/S0921-8890(02)00372-X

11. Forkan R, Pumper B, Smyth N, Wirkkala H, Ciol MA, ShumwayCook A (2006) Exercise adherence following physical therapy intervention in older adults with impaired balance. Phys Therapy 86(3):401-410

12. Forlizzi J, DiSalvo C, Gemperle F (2004) Assistive robotics and an ecology of elders living independently in their homes. Hum Comput Interact 19(1):25-59. https://doi.org/10.1207/ s15327051hci1901\&2_3

13. Gadde P, Kharrazi H, Patel H, MacDorman KF (2011) Toward monitoring and increasing exercise adherence in older adults by robotic intervention: a proof of concept study. J Robot 2011:e438e514. https://doi.org/10.1155/2011/438514

14. Gale NK, Heath G, Cameron E, Rashid S, Redwood S (2013) Using the framework method for the analysis of qualitative data in multi-disciplinary health research. BMC Med Res Methodol 13:117. https://doi.org/10.1186/1471-2288-13-117

15. Gockley R, Bruce A, Forlizzi J, Michalowski M, Mundell A, Rosenthal S, Sellner B, Simmons R, Snipes K, Schultz AC, Wang J (2005) Designing robots for long-term social interaction. In: 2005 IEEE/RSJ international conference on intelligent robots and systems, pp 1338-1343. https://doi.org/10.1109/IROS.2005.1545303

16. Gockley R, Mataric MJ (2006) Encouraging physical therapy compliance with a hands-off mobile robot. In: Proceedings of the 1st ACM SIGCHI/SIGART conference on human-robot interaction, pp 150-155. ACM

17. Green A, Huttenrauch H, Norman M, Oestreicher L, Eklundh KS (2000) User centered design for intelligent service robots. In: Proceedings 9th IEEE international workshop on robot and human interactive communication. IEEE RO-MAN 2000 (Cat. No.00TH8499), pp 161-166. https://doi.org/10.1109/ROMAN. 2000.892488

18. Gross HM, Schroeter C, Mueller S, Volkhardt M, Einhorn E, Bley A, Langner T, Martin C, Merten M (2011) I'll keep an eye on you: Home robot companion for elderly people with cognitive impairment. In: 2011 IEEE international conference on, systems, man, and cybernetics (SMC), pp 2481-2488. IEEE

19. Jenkins S, Draper H (2015) Care, monitoring, and companionship: views on care robots from older people and their carers. Int J Soc Robot 7(5):673-683

20. Jones S (1992) Was there a Hawthorne effect? Am J Sociol 98(3):451-468. https://doi.org/10.1086/230046

21. Kidd CD, Taggart W, Turkle S (2006) A sociable robot to encourage social interaction among the elderly. In: Proceedings 2006 IEEE international conference on robotics and automation, 2006. ICRA 2006, pp 3972-3976. IEEE

22. Lee HR, Sabanovic S, Chang WL, Nagata S, Piatt J, Bennett C, Hakken D (2017) Steps toward participatory design of social robots: mutual learning with older adults with depression. In: Proceedings of the 2017 ACM/IEEE international conference on human-robot interaction, HRI '17, pp 244-253. ACM, New York, NY, USA. https://doi.org/10.1145/2909824.3020237

23. Leite I, Pereira A, Castellano G, Mascarenhas S, Martinho C, Paiva A (2011) Modelling empathy in social robotic companions. In: Advances in user modeling, lecture notes in computer science, pp 135-147. Springer, Berlin. https://doi.org/10.1007/978-3-64228509-7 14

24. Louie WYG, Li J, Vaquero T, Nejat G (2014) A focus group study on the design considerations and impressions of a socially assistive robot for long-term care. In: The 23rd IEEE international symposium on robot and human interactive communication, pp 237-242. https://doi.org/10.1109/ROMAN.2014.6926259

25. Montemerlo M, Pineau J, Roy N, Thrun S, Verma V (2002) Experiences with a mobile robotic guide for the elderly. In: AAAI/IAAI, pp 587-592

26. Nani M, Caleb-Solly P, Dogramadzi S, Fear T, van den Heuvel H (2010) MOBISERV: an integrated intelligent home environment for the provision of health, nutrition and mobility services to the elderly. http://eprints.uwe.ac.uk/16102/1/MOBISERV_ WorkshopPaper.pdf. Accessed 1 Oct 2015

27. O'Shea SD, Taylor NF, Paratz JD (2007) But watch out for the weather: factors affecting adherence to progressive resistance exercise for persons with COPD. J Cardiopulm Rehabilit Prev 27(3):166-174. https://doi.org/10.1097/01.HCR. 0000270686.78763.c8 quiz 175-176

28. Sabanovic S (2010) Robots in society, society in robots. Int J Soc Robot 2(4):439-450. https://doi.org/10.1007/s12369-010-0066-7

29. Sabanovic S, Meisner E, Caporael LR, Isler V, Trinkle J (2009) Outside-in design for interdisciplinary HRI research. https://www. aaai.org/Papers/Symposia/Spring/2009/SS-09-03/SS09-03-006. pdf. Accessed 1 Jan 2018

30. Sabelli AM, Kanda T, Hagita N (2011) A conversational robot in an elderly care center: an ethnographic study. In: 2011 6th ACM/IEEE international conference on human-robot interaction (HRI), pp 3744. https://doi.org/10.1145/1957656.1957669

31. Sussenbach L, Riether N, Schneider S, Berger I, Kummert F, Lutkebohle I, Pitsch K (2014) A robot as fitness companion: towards an interactive action-based motivation model. In: The 23rd IEEE international symposium on robot and human interactive communication, pp 286-293. https://doi.org/10.1109/ROMAN.2014. 6926267

32. Swift-Spong K, Short E, Wade E, Mataric MJ (2015) Effects of comparative feedback from a socially assistive robot on selfefficacy in post-stroke rehabilitation 2015 IEEE International Conference on Rehabilitation Robotics (ICORR). IEEE

33. Tapus A, Mataric MJ (2008) Socially assistive robots: The link between personality, empathy, physiological signals, and task performance. In: AAAI spring symposium: emotion, personality, and social behavior, pp 133-140

34. Tapus A, Tapus C, Mataric M (2009) The role of physical embodiment of a therapist robot for individuals with cognitive impairments. In: The 18th IEEE international symposium on robot and human interactive communication, 2009. RO-MAN 2009, pp 103-107. IEEE

35. Tapus A, Tapus C, Mataric MJ (2009) The use of socially assistive robots in the design of intelligent cognitive therapies for people with dementia. In: 2009 IEEE international conference on rehabilitation robotics, pp 924-929. https://doi.org/10.1109/ICORR.2009. 5209501 
36. Venkatesh V, Davis FD (2000) A theoretical extension of the technology acceptance model: four longitudinal field studies. Manag Sci 46(2):186-204. https://doi.org/10.1287/mnsc.46.2.186.11926

37. Venkatesh V, Morris MG, Davis GB, Davis FD (2003) User acceptance of information technology: toward a unified view. MIS Q 27(3):425-478

38. Visser M, Brychta RJ, Chen KY, Koster A (2014) Self-reported adherence to the physical activity recommendation and determinants of misperception in older adults. J Aging Phys Act 22(2):226-234. https://doi.org/10.1123/japa.2012-0219

39. Vries RAJd, Truong KP, Zaga C, Li J, Evers V (2017) A word of advice: how to tailor motivational text messages based on behavior change theory to personality and gender. Pers Ubiquitous Comput 21(4):675-687. https://doi.org/10.1007/s00779-017-1025-1

40. Wilk R, Johnson MJ (2014) Usability feedback of patients and therapists on a conceptual mobile service robot for inpatient and home-based stroke rehabilitation. In: 5th IEEE RAS/EMBS international conference on biomedical robotics and biomechatronics, pp 438-443. https://doi.org/10.1109/BIOROB.2014.6913816

41. Williams A, Stephens R, McKnight T, Dodd S (1991) Factors affecting adherence of end-stage renal disease patients to an exercise programme. Br J Sports Med 25(2):90-93. https://doi.org/10. 1136/bjsm.25.2.90

42. Williams R, Edge D (1996) The social shaping of technology. Res Policy 25(6):865-899. https://doi.org/10.1016/00487333(96)00885-2

43. Winkle K, Caleb-Solly P, Turton A, Bremner P (2018) Social robots for engagement in rehabilitative therapies: design implications from a study with therapists. In: Proceedings of the 2018 ACM/IEEE international conference on human-robot interaction, HRI '18, pp 289-297. ACM, New York, NY, USA. https://doi.org/ $10.1145 / 3171221.3171273$

44. Wu YH, Fassert C, Rigaud AS (2012) Designing robots for the elderly: appearance issue and beyond. Arch Gerontol Geriatr 54(1):121-126. https://doi.org/10.1016/j.archger.2011.02.003

Publisher's Note Springer Nature remains neutral with regard to jurisdictional claims in published maps and institutional affiliations.

Katie Winkle is a PhD student in the EPSRC Centre for Doctoral Training in Future Autonomous and Robotic Systems (FARSCOPE) at the Bristol Robotics Laboratory. Her research is focused on social influence in human robot interaction, which she is currently exploring in the context of socially assistive robotics e.g. for use in rehabilitative therapies.
Praminda Caleb-Solly is Professor of Assistive Robotics and Intelligent Health Technologies at the Bristol Robotics Laboratory. She is also part of the University's Health Tech Hub which helps businesses to develop new technology solutions which promote health and wellbeing. From 2014 to 2018, she was also Head of Electronics and Computer Systems at Designability, a not-for-profit SME who design Assistive Technology. She holds a BEng in Electronic Systems Engineering, MSc in Biomedical Instrumentation Engineering and a PhD in Interactive Evolutionary Computation. Her recent portfolio of Innovate UK, EPSRC, AHRC and EC funded projects include designing and evaluating socially and physically assistive robotics and IoT sensor-based intelligent technology to support older adults with ageing-related impairments, working in partnership with care providers. She is currently running an Assistive Robotics in Healthcare Demonstrator as part of the Assuring Autonomy International programme. Her research covers safe, accessible and collaborative human-robot Interaction, sensing and intelligent activity recognition, adaptive machine learning systems and multimodal interfaces for people with accessibility needs. She has over 60 refereed journal and conference publications and co-authored the EPSRC UK-RAS white paper on Robotics in Social Care.

Dr. Ailie Turton is an Occupational Therapist and Senior Lecturer in the Dept of Allied Health Professions at the University of the West of England, Bristol. Her research spans rehabilitation sciences, neurosciences and development of new treatments and technologies for rehabilitation and assisted living. Using mixed methods for developing and evaluating complex interventions, the primary aim of her research is to improve recovery of function for people with stroke, to enable them to participate fully in everyday life activities. Current work related to rehabilitation robotics at Bristol Robotics Laboratory includes Wearable Soft Robotics for Independent Living (funded by the Engineering and Physical Sciences Research Council, UK); Ailie is lead for user perceptions and specifications for the devices.

Paul Bremner received a BSc in Robotic and Electronic Systems Engineering from the University of Salford in 2003. He received an MSc in Advanced Technologies in Electronics and a $\mathrm{PhD}$ in Human-Robot interaction from the University of the West of England in 2005 and 2010 respectively. Since 2010 he has worked at the University of the West of England on a number of projects, first as a research associate then as a research fellow, where he is currently employed on the EPSRC-funded programme grant Robots for Nuclear Environments. His research interests include human-robot interaction, multi-modal communication, tele-presence, machine learning and robot ethics. 